\title{
Design, Implementation and Simulation of an Experimental Multi-Camera Imaging System for Terrestrial and Multi-Purpose Mobile Mapping Platforms: A Case Study
}

\author{
Karim Hammoudi ${ }^{1,2, a}$, John McDonald ${ }^{1, b}$ \\ ${ }^{1}$ Department of Computer Science, National University of Ireland Maynooth, Co. Kildare, Ireland \\ ${ }^{2}$ Lab. MATIS, Univ. Paris-Est, Institut National de l'Information Géographique et Forestière, France \\ akarim.hammoudi@cs.nuim.ie, bjohnmcd@cs.nuim.ie
}

Keywords: machine vision, imagery infrastructure, mechanical implementation, geo-engineering.

\begin{abstract}
Mobile Mapping Systems (MMS's) are powerful tools for rapidly and massively collecting imagery in various environments (e.g., rural, semi-urban, urban). In particular, the data collected at terrestrial level can be exploited to complement aerial acquisitions for extending GIS databases, visualizing and modeling environments and studying their morphogenesis over time. Hence, the development of mobile mapping platforms is a topic of great interest for many mapping agencies and surveying companies. Moreover, the experimental imaging systems of mapping vehicles are equipped with varied infrastructures in part resulting from the wide range of targeted applications. Determining a detailed design procedure for such imaging systems is of critical importance, and can be both arduous and time-consuming. Although commercial imaging systems can be exploited directly they are often pre-configured for specific applications. For these reasons, we propose a case study that deals with the development of an orientable and scalable imaging system. In particular, this paper focusses on the primary stage of the mechanical implementation of a multi-camera infrastructure.
\end{abstract}

\section{Introduction and Motivation}

Nowadays, Mobile Mapping Systems are actively developed in order to massively collect multisource imagery at street level for the generation of future urban GIS databases. From the early $90^{\prime} s$, important efforts have been led in the development of operational MMS's (e.g.; $[1,2])$ and one can observe meaningful progression for the combined use of various sensors and cameras (e.g.; [3, 4]).

Many imaging systems are composed of stereoscopic and/or panoramic cameras (e.g., see Figure 1(a)) and some emerging MMS's also integrate specific cameras that can provide detph or thermographic information corresponding to the acquired 2D images. The huge amount of collected heterogeneous image data constitutes raw resources for image processing, computer vision and computer graphics communities that supports the development of research in a variety of topics related to the earth mapping.

Specific examples of such research include the utilisation of MMS imagery for the inspection of road surfaces (e.g., [5]), the identification of road marks (e.g., [6]), the analysis of heat loss for building facades (e.g., [7]) and the generation of textured 3D models for building facades (e.g., [8]), to name a few. Moreover, the image data are also more directly employed in applications related to the large-scale visualization of street scenes (e.g., image mosaicing). Notably, one can mention popular applications such as Microsoft Street Slide [9] or Google Street View [4]. Hence, the development of operational MMS's for collecting image data on a massive scale, and the related research and application in the large field of geomatics, is of great interest for many governmental mapping agencies and land surveying companies.

As previously mentioned, producing a detailed design procedure for creating such a system can be an arduous and time-consuming task. For this reason, we provide here a technical document that has been elaborated in cooperation with machine vision researchers, mechanical engineering experts and camera suppliers towards supporting external stakeholders engaging in analogous developments and thus facilitating the creation of multi-camera imaging systems. 


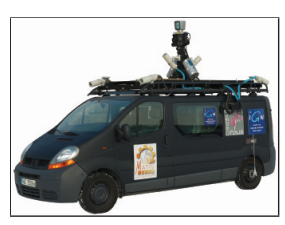

(a)

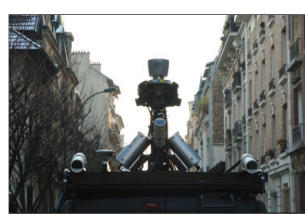

(b)

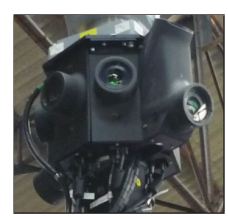

(c)

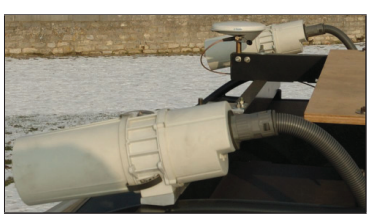

(d)

Fig. 1: Multi-view camera system mounted on a van platform.

\section{Related Work}

As mentioned above, in this paper our interest is specifically in creating camera-based imaging systems. Indeed, camera-based systems can be a cost-effective solution for investigating research in all the areas previously mentioned. However, we emphasize that many MMS's also integrate complementary mapping devices such as laser sensors or GPR's (Ground Penetrating Radars; e.g., refer to [3] and [5], respectively). Below, we describe MMS's that are equipped with various roof-mounted multi-camera imaging systems, including commercial- and/or research-orientated platforms.

Topcon and Mitsubishi propose rack-mounted versions with multi-view camera systems (e.g., IP-S Lite and MMS-X respectively). These systems are both equipped with Point Grey Ladybug ${ }^{\mathbb{R}}$ cameras, a model which is widely used in the MMS industry (e.g., see the pentagonal-shaped red box in the figures in [10]). More precisely, these cameras integrate five lens that are located shoulder to shoulder as well as one central lens facing the sky. These imaging systems are very compact and complete in the sense that $i$ ) they are integrated in a single-camera setup mounted at the top of a column, and, $i$ ) this camera directly outputs a spherical visualization of the scene (i.e. all mosaic processing is handled internally by the camera). Such compact multi-view systems are actively exploited for generating and maintaining up-to-date GIS databases of urban environments and have also been used for rapidly and efficiently generating diagnostic maps in emergency mapping (e.g.; maps of disaster areas).

The Earthmine MMS (e.g; see [3]) is equipped with a vision system for digitizing streets that exploits technologies developed by the NASA's Jet Propulsion Laboratory such as those used on the Mars Rover. This system is composed of a central vertical mast that is equipped with two camera sets located at the top and bottom of the mast. Each camera set is composed of four independent cameras that are horizontally oriented in cardinal or ordinal directions. Hence each set of four cameras provides a panoramic camera system. Hence, the overall multi-view system constitutes a customized stereo panoramic system. The combined use of these cameras can provide high resolution panoramic images with the 3D point coordinates associated to each pixel. Moreover, this system presents ergonomic aspects. The camera mast permits reconfiguration of the overall imaging system, including adjusting the camera locations (e.g., stereoscopic camera baseline) or adding devices along the vertical axis. The interior of the mast (hollow mast) can be exploited for protecting and concealing device cables and the mast can be positioned horizontally when not in use. Furthermore, the overall system is installed on a modular rack that has the advantage of being mountable on almost any vehicle as well as on pedestrian platforms. In this way, the camera system is designed for collecting imagery in large-scale and dense environments and covering entire metropolitan areas.

At the Institut Géographique National (French national mapping agency), they have developed an MMS named Stereopolis (see Figure 1(a)) which also utilises a central mast which is installed onto a vehicle platform covering the roof surface (see Figure 1(b)). The MMS masthead (Figure 1(c)) is composed of eight cameras oriented horizontally in cardinal and ordinal directions that provide an omnidirectional vision system. Moreover, two additional cameras are laterally located in the direction of the top of the street facades (i.e. towards the roof level). This set of cameras is enclosed in a carbon protective housing. A matte black color is used throughout the vehicle and system parts for reducing the reflectance effects in the collected imagery. The vehicle is also equipped with two stereoscopic acquisition systems that are located at the front and rear areas of the vehicle and oriented obliquely 


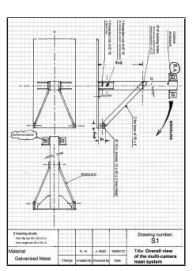

(a)

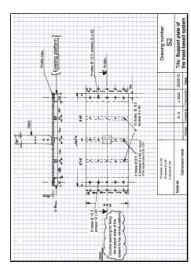

(b)

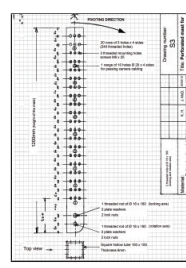

(c)

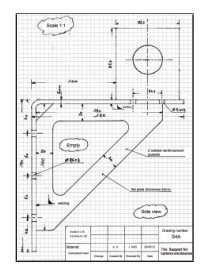

(d)

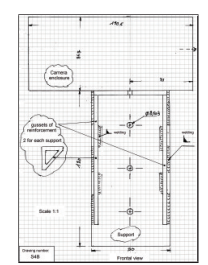

(e)

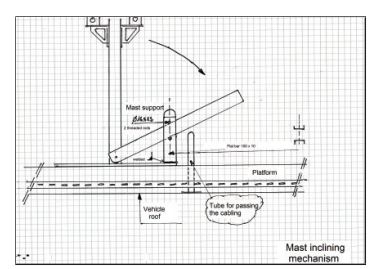

(f)

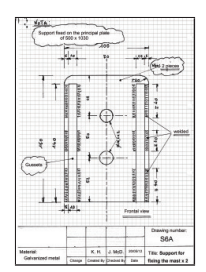

(g)

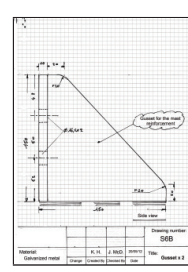

(h)

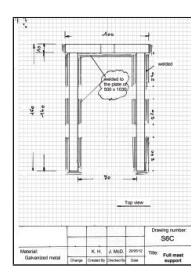

(i)

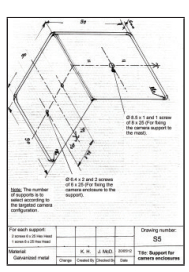

(j)

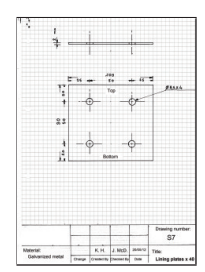

(k)

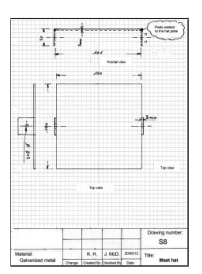

(1)

Fig. 2: Set of hand-drawn engineering drawings elaborated for the design of a multi-view mast system. Figures 2(a) to 2(f) and Figures 2 (g) to 2(1) illustrate detailed views of major elements for the mast mounting and mast accessories, respectively. We point out the fact that the complete set of original drawings presented in this study (A4 page format) can be downloaded from http://www. cs.nuim.ie/research/vision/data/ optirob2013/.

in direction of the road (see Figure 1(d)). Hence, the mapping system is relatively multi-purpose. The collected imagery are actively exploited for mapping various objects in urban environments with a high accuracy (e.g., for metrology applications). Further information on this camera system as well as complete procedure to accurately calibrate a multi-camera mobile mapping system are presented in $[11,12]$.

Google has developed some MMS fleets with mast-based configurations for collecting street images that has been deployed worldwide. Notably, we can observed in the literature a fleet version of Google Street View Cars (GSV cars) that are equipped with an imposing mast mounted onto a vehicle rack. This mast is foldable and is laterally equipped with two linear actuators as well as two bracing struts for maintaining and stabilizing the mast in vertical position. This masthead is equipped with a set of nine cameras that produces street view panoramas. Further information on this camera system and panoramic-based applications are given in [13]. Also, some works describe design resources and practical instructions for creating GSV-like multi-camera systems. One can notably mention a study of an artistic group presented in [14] for replicating $\boldsymbol{a}$ GSV car as well as a study presented in [15] for creating a DIY Street-View Camera system by exploiting conventional webcams (low-cost system).

\section{Proposed Design and Implementation}

In this section, we present a design study for creating an experimental multi-camera mast system for roof-mounted vehicle platforms for the development of multi-purpose mobile mapping systems. In particular, we are focused on the primary stage of the system design that lies in the mechanical implementation of the multi-camera infrastructure. This study aims at producing a flexible and scalable system in such a way that cameras can be located and oriented in order to acquire various object categories (e.g.; parked vehicles, street signs, facades). Moreover, for each targeted category, it should be possible to operate at accurate adjustments of camera orientations. Notably, experiments from [16] show that the camera orientations have to be determined according to the type of targeted objects in order to reduce inaccuracies in the 3D measurements. Furthermore it should be possible to replace or add cameras, thereby permitting the system configuration to be altered according to user's needs. Furthermore, we aim to minimize the complexity of system components and the volume of required raw materials and as a consequence reduce both the fabrication cost and time, respectively.

A global view of the designed multi-camera mast system is illustrated in Figure 2(a). The presented system is composed of a square hollow mast that is mounted on a rectangular supporting plate 
and affixed by two lateral supports. The mast can include a set of digital cameras mounted on orientable supports and is designed to be collapsible. As such, acquisitions of street images are carried out with the mast in vertical position (on-line configuration) and then the mast is folded down in oblique position after use (off-line configuration). This system can be created from carbon steel or stainless steel (the latter having a high resistance to corrosion and oxidation). Detailed information of the system components are given below.

The rectangular plate that supports the mast is illustrated in Figure 2(b). The dimension of this plate should be adjusted according to the model of the intended platform. Basically, the plate can be fixed to various grid-based platforms from counter-drilled holes (see Figure 2(b)). Moreover, small vertical pads can be welded under the supporting plate in order to avoid shearing of the fixations during the vehicle motion. Furthermore, an hole can be added at the center of the plate to allow camera cables to be passed from underneath to the interior of the mast. According to the platform structure and the resistance of materials, openings can added in the supporting plate in order to lighten the overall system.

Figure 2(c) depicts specific details of the column mast. The mast is composed of an hollow tube that is perforated at each side with ranges of holes with different diameters. The first range of holes are small fixing holes that can be employed for positioning and adjusting camera locations (e.g., stereoscopic camera baseline) and camera support orientations (sloping angle). Also, these ranges can be exploited for fixing other mapping devices along the vertical axis. Second ranges are large holes that can be employed for accessing camera cabling passing at the interior of the mast. These fixing holes can be either holes threaded next to the mast or conventional holes directly equipped with internally threaded fasteners (tubular rivets with internal threads). The later solution being more economical. In conjunction with the above, the mast tube has been curved at its base in order to allow the column rotation in on-line and off-line configurations.

As previously mentioned, the mast is maintained at its basis by lateral fixing supports that are welded to the mast supporting plate (see Figures 2(g) and 2(a)). In order to ensure the rigidity of the mast during vehicle displacements, the fixing supports (at mast/plate junctions) have been reinforced at each side by including pairs of welded brackets (see Figures 2(h) and 2(i)). Furthermore, lateral bracing struts (stabilizer bars) have also been added such as shown in the overall scheme (Figure 2(a)). Notably, the system rigidity is necessary for determining accurate camera poses relative to the platform (system calibration).

Figures 2(d) and 2(e) show schematics for the orientable camera supports in frontal and lateral view, respectively. The camera supports integrate a set of gussets (reinforcement brackets) including openings for supporting the camera enclosures. Basic camera supports such as drawn in Figure 2(j) can also be used for small enclosures. These supports can integrate angle graduations for tuning the sloping angle of cameras. According to the user's needs, these two models of camera supports can be fixed to the mast at each side and with various locations and orientations. Camera suppliers can also propose camera enclosures equipped with their 3D mounts (e.g., APG (http://apgvision.com/), Ganz (http: / / computarganz.com/) or autoVimation (http://www. autovimation.com/)).

Additionally, the mast system can be completed with some accessories. In particular, Figure 2(k) illustrates a model of lining plates that is designed for the camouflage of the unused fixing holes. Figure 2(1) illustrates the mast hat that is composed of a square plate with two welded lugs. The surface of the mast hat, the remaining mast fixing holes and the surface of the mast supporting plate can be exploited for installing additional mapping devices (e.g., GPS and laser sensors). Finally, Figure 2(f) shows the mast folded down and secured in its cradle. Here, the cradle consists of two rear lateral plates positioned vertically and secured by two traversing rods (one for supporting the mast and the other for immobilizing the mast). A cradle can also be created by using an incised vertical plate. 


\begin{tabular}{|r|c|c|c|c|c|c|}
\hline Major Components of the System & CS $^{1}$ Material & CA $^{2}$ & Quantity & Dimensions $(\mathrm{mm})$ & Metal Thickness $(\mathrm{mm})$ & Weight $(\mathrm{Kg})$ \\
\hline \hline Support plate for the mast (Fig.2(b)) & Plate & $\boldsymbol{X}$ & 1 & $1030 \times 500$ & 10 & $\simeq 40$ \\
\hline Mast (Fig.2(c)) & Square hollow tube & $\checkmark$ & 1 & $150 \times 150 \times 1350$ & 5 & $\simeq 31$ \\
\hline Supports fixing the mast (Fig.2(g)) & Plate & $\boldsymbol{X}$ & 2 & $150 \times 150$ & 10 & $\simeq 4$ \\
\hline Brackets fixing the mast (Fig.2(h)) & Plate & $\boldsymbol{X}$ & 4 & $150 \times 150$ & 10 & $\simeq 8$ \\
\hline Supports for camera (Fig.2(d), Fig.2(e)) & Flat bar & $\checkmark$ & 6 & $100 \times 100$ & $8($ or 10$)$ & $\simeq 7.5$ \\
\hline Bracing struts for stabilizing the mast & Flat bar & $\checkmark$ & 2 & $50 \times 1000$ & \multicolumn{1}{c|}{} \\
\hline Cradle of the mast & Plate & $\boldsymbol{X}$ & 1 & $250 \times 500$ & 10 & $\simeq 9$ \\
\hline \hline Overall System (Fig.2(a)) & - & - & - & - & - & $\simeq 106$ \\
\hline
\end{tabular}

Table 1: Inventory describing the material required for creating the major components of the designed multi-view camera system - BOM (Bill Of Materials) and the corresponding set of technical characteristics. ${ }^{1} \mathrm{CS}$ : Carbon Steel (Density $\simeq 7.85 \mathrm{~g} / \mathrm{cm}^{3}$ ). ${ }^{2} \mathrm{CA}$ : Commercially Available. The surface of a single standard plate of dimension $D=2000 \mathrm{~mm} \times 1000 \mathrm{~mm}$ and thickness $T=10 \mathrm{~mm}$ can be used for creating the support plate of the mast (Figure 2(b)), the supports for fixing the mast (Figure 2(g)), the reinforcement brackets for fixing the mast (Figure 2(h)) and the support for maintaining the mast in the folded down position (i.e., mast cradle illustrated in Figure 2(f)). A standard square hollow tube of dimension $D=150 \mathrm{~mm} \times 150 \mathrm{~mm} \times 6000 \mathrm{~mm}$ and thickness $T=5 \mathrm{~mm}$ can be directly employed for creating the mast (Figure 2(c))

\section{Application and Evaluation}

In this Section, we describe required resources for creating the proposed system for a vehicle platform and we evaluate the applicability of the proposed design in terms of budget, realization and usage.

\section{Experimental Setup}

\begin{tabular}{|c|c|c|}
\hline \begin{tabular}{|ll} 
Raw resources & Type \\
\end{tabular} & Carbon Steel & Stainless Steel \\
\hline Principal plate $^{\perp}$ & $€ 190$ & $€ 842$ \\
\hline Square hollow tube ${ }^{1}$ & $€ 117$ & $€ 540$ \\
\hline Consumables $^{2}$ & $€ 114$ & $€ 331$ \\
\hline Labour $^{2}$ & $€ 4000$ & $€ 4000$ \\
\hline Total cost ${ }^{2}$ & $€ 4482$ & $€ 5989$ \\
\hline
\end{tabular}

Table 2: Estimated cost of the multi-camera system infrastructure. The prefabrication and assembly time for creating the designed system is estimated to 80 person hours. Note that this time does not include for installation and cabling. The labour cost is calculated on a rate of $€ 50$ per hour. The mentioned total cost is estimated by considering $R P=1.2 \times P P$ where ${ }^{1} P P$ and ${ }^{2} R P$ corresponds to the Purchase Price and Resale Price, respectively. These estimates were gathered via quotes from a leading engineering company in Northern France in August 2012.
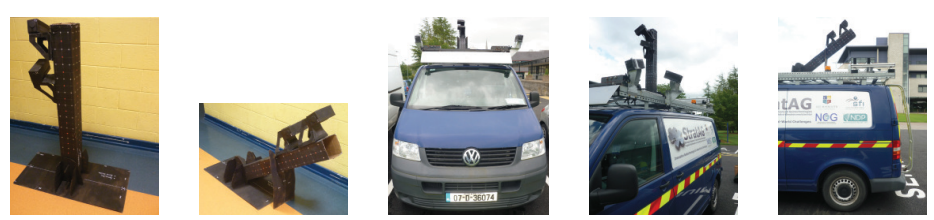

Fig. 3: (indoor) Created real-scale 3D mock-up of the mast. The mast components have been produced and assembled from the elaborated engineering drawings. (outdoor) Multi-view camera mast system installed on the NUIM MMS. Central and right images show the multi-camera mast system with additional peripheral cameras (on-line configuration) and the mast folded down in its cradle (off-line configuration), respectively. Notably, we can observe the mast held in position by the two metal stems, i.e. providing the axis used for the mast rotation and vertical immobilization. Also shown in the indoor figures, the reference points correspond to the mast perforation locations. The rectangular shaped-boxes mounted on the mast are replicas of commercialised $\boldsymbol{A P G}$ 30D-AD camera housings $D=76.2 \mathrm{~mm} \times 85.7 \mathrm{~mm} \times 190.5 \mathrm{~mm}$ - the waterproof camera housings which will be used in the final NUIM MMS. The illustrated results clearly demonstrate the applicability of the proposed design study for creating the multi-camera mast system.

Table (1) provides an inventory of the material required for creating the principal components of the designed multi-view camera mast. More precisely, we have established the BOM (Bill Of Materials) including the technical characteristics. A number of the listed components that can be manufactured from commercially available materials with standard dimensions and optimisations for the system fabrication have been described. An estimated total cost of the presented system is detailed in Table (2) according to the selected steel type.

\section{Created Real-scale 3D Model and Usage Scenarios}

As a final stage in the evaluation we constructed a full-scale prototype of the proposed multicamera mast system design. This prototype aims at assessing the range of locations and viewing angles provided by the mast when installed on the National University of Ireland Maynooth (NUIM) MMS. The resulting prototype (shown in Figure 3) has been completely created from painted semirigid paper-boards providing adequate rigidity for the assessment exercise. The created camera mast $(H=1.35 \mathrm{~m})$ and scenarios for on-line and off-line configurations are illustrated. Here, the mast is equipped with two cameras that are oriented with an angular sloping of $+40^{\circ}$ and $-10^{\circ}$ with respect to the horizontal plane for acquiring images at the top and bottom of the facade, respectively. The height of the vehicle is $2.25 \mathrm{~m}$ and reaches $3.60 \mathrm{~m}$ with the mast camera installed in the vertical position. Also, this scenario includes additional peripheral cameras that are mounted frontally and laterally for acquiring images of road surfaces and street-side urban objects, respectively. These cameras are mounted on a single vehicle side in the sense that the other side can be completed for roads having a two-way traffic flow. However, many scenarios can be envisaged according to the developed mapping activities. 


\section{Conclusion and Discussion}

This paper provides an overview of design characteristics and usages of multi-view camera systems for some operational mobile mapping vehicles. An experimental study has been produced in cooperation with machine vision researchers, mechanical engineering experts and camera suppliers towards supporting external stakeholders engaged in the development of experimental multi-camera imaging systems for use in MMS platforms. In particular, practical and methodological instructions have been provided for creating a flexible and scalable system in term of camera configurations and for reducing the fabrication complexity, time and cost. The paper is accompanied by a full set of detailed design drawings (available online) as well as a detailed inventory of system components required for creating the multi-camera imaging mast. The applicability of the proposed design has been demonstrated through the creation of a full scale 3D model and assessment exercises.

We believe the design is extensible with the potential for additonal components to be integrated according to the needs and resources of the user. For example, practical mechanisms such as a telescopic mast or powered actuators for increased automation could easily incorporated into the desing. At NUIM, we have adopted a two-step implementation/investment strategy. In a first time, some of the designed housing supports have been produced for rapidly creating frontal and back stereoscopic camera systems for the NUIM MMS. In a second time, we expect to produce the remaining central parts of the designed camera mast.

Acknowledgements: The research was funded by a Strategic Research Cluster grant (07/SRC/I1168) by Science Foundation Ireland under the National Development Plan. The authors gratefully acknowledge this support. They also thank Larbi Hammoudi, awarded as "One of the Best Craftsmen of France" in the field of metallurgy, for his advice.

\section{References}

[1] Novak Kurt. The Ohio State University Highway Mapping System: The Stereo Vision System Component. In Proc. Annual Meeting of the Institute of Navigation, pp. 121-124, 1991.

[2] Ellum, C.M. and El-Sheimy, N. Land-based mobile mapping systems. In Proc. Photogrammetric Engineering and Remote Sensing, Vol. 68, Numero 1, pp. 13-17, 2002.

[3] Petrie, G. Mobile Mapping Systems: An introduction to the technology. In GeoInformatics, Vol. 13, Issue 1, pp. 32-43, 2010.

[4] D. Anguelov, C. Dulong, D. Filip, C. Frueh, S. Lafon, R. Lyon, A. Ogale, L. Vincent, J. Weaver. Google street view: Capturing the world at street level. In IEEE Computer, Vol. 43, pp. 32-38, 2010.

[5] S. Chambon, J.-M. Moliard. Automatic road pavement assessment with image processing: review and comparison. In International Journal of Geophysics, Vol. 2011, 20p., 2011.

[6] B. Soheilian, N. Paparoditis, D. Boldo. 3D road marking reconstruction from street-level calibrated stereo pairs. In ISPRS Journal of Photogrammetry and Remote Sensing, Vol. 65, I-4, pp. 347-359, 2010.

[7] J. Hopper, J. R. Littlewood, T. Taylor, J. A.M. Counsell, A. M. Thomas, G. Karani, A. Geens, N. I. Evans. Assessing retrofitted external wall insulation using infrared thermography. In Structural Survey, Vol. 30, Issue 3, pp. 245-266, 2012.

[8] K. Hammoudi; F. Dornaika, B. Soheilian, B. Vallet, J. McDonald, N. Paparoditis. Recovering occlusion-free textured 3D maps of urban facades by a synergistic use of terrestrial images, 3D point clouds and area-based information. In Procedia Engineering, Vol. 41, Issue C, pp. 971-980, 2012.

[9] J. Kopf, B. Chen, R. Szeliski, M. Cohen. Street Slide: Browsing Street Level Imagery. In ACM Transactions on Graphics, Vol. 29, Number 4, pp.96:1-96:8, 2010.

[10] T. Chen, K. Yamamoto, S. Chhatkuli, H. Shimamura. Panoramic epipolar image generation for mobile mapping system. In ISPRS Annals, Vol. 39-B5, pp. 459-464, 2012.

[11] N. Paparoditis, J.-P. Papelard, B. Cannelle, A. Devaux, B. Soheilian, N. David, E. Houzay. Stereopolis II: A multi-purpose and multi-sensor 3D mobile mapping system for street visualization and 3D metrology. In Revue Française de Photogrammétrie et de Télédétection, Number 200, pp. 69-79, 2012.

[12] B. Cannelle, N. Paparoditis, M. Pierrot-Deseilligny and J.-P. Papelard. Off-line vs. on-line calibration of a panoramic-based mobile mapping system. In ISPRS Annals, Vol. I-3, pp. 31-36, 2012.

[13] Victor J.D. Tsai and Chung-Ting Chang. Feature positioning on Google street view panoramas. In ISPRS Annals, Vol. I-4, pp. 305-309, 2012.

[14] FATlab members. How to build a fake Google street view car. Transmediale, 2010. http://fffff.at/ google-street-view-car/ [accessed 29 January 2013]

[15] Ragsdale, R.D. DIY Street-View Camera. In IEEE Spectrum, Vol. 46, Issue 10, pp. 20-21, 2009.

[16] S-Y Lee, K-H Choi, I-H Joo, S-I Cho, J-H Park. Design and implementation of 4S-Van: A Mobile Mapping System. In ETRI Journal, Vol. 28, Number 3, 2006. 